\title{
Envelope/phase delays correction in an EER radio architecture
}

\author{
Jean-François BERCHER and Corinne BERLAND \\ ESYCOM / ESIEE \\ Noisy-le-Grand, France \\ jf.bercher@esiee.fr, c.berland@esiee.fr
}

\begin{abstract}
In this paper we consider the sensitivity of a transmitter based on EER radio architecture to time mismatches between phase and envelope. We propose an adaptive algorithm to compensate the delays and demonstrate the interest of this approach.
\end{abstract}

\section{INTRODUCTION}

Envelope Elimination and restoration (EER) was developed by Kahn in 1957 [1] and appears to be a solution to deal with complex modulation scheme [2]. Indeed, efficient modulations present significant envelope variation as for example the OFDM one (Orthogonal Frequency Division Multiplex) used for wireless LAN systems. The PAPR (Power to Average Peak Ratio) for a 16QAM 64 sub carriers OFDM is up to $18 \mathrm{~dB}$. For a less complex modulation, such as a classical 16 QAM with a raised cosine filter, the PAPR is lowered to $6 \mathrm{~dB}$. In order to avoid distortions which would impact both EVM (Error Vector Magnitude) and output spectrum, linearization method or highly linear amplification is necessary. The EER principle relies on the separation of the phase and envelope signal as presented on Fig.1.

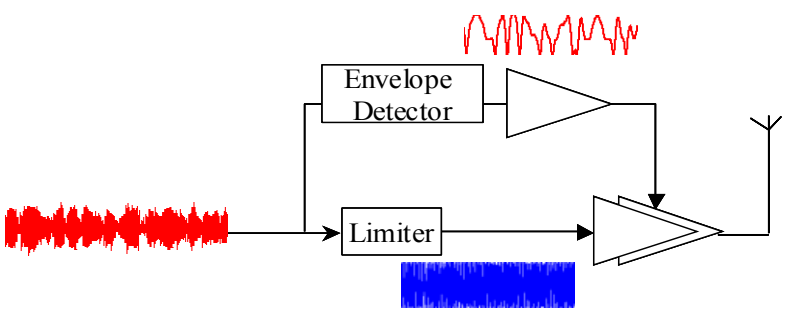

Figure 1. EER principle

The nonlinear input/output characteristics of the PA (power amplifier) generate intermodulation terms when the input signal presents envelope variation. With a constant envelope phase modulated signal, this phenomena is avoided, enabling linear amplification. As for the envelope variation, it is reintroduced through the supply voltage of the PA last stage. In the first EER solution, the phase signal was obtained after a limiting action on the modulated signal and the envelope one using a simple envelope detector. The actual evolution of this architecture is the digital generation of both signals.

Few studies were presented concerning the specification of such a solution applied to complex modulations [3]. This paper concerns the sensibility analysis of the EER on the following points: signals bandwidth, impact of the envelope restoration, impact of the desynchronisation between the two path, etc... These studies pointed up that the critical specification is the synchronization of signals. Performances to achieve are so tight that a synchronization algorithm has to be implemented.

\section{IMPACT OF THE DESYNCRHONISATION OF PHASE AND ENVELOPE SIGNALS}

Transmitter performances are usually given in term of output spectrum and EVM. As performance reference, we will take for example the 802.11a standard, where EVM is specified for a 16QAM 52 sub-carriers OFDM to $11.22 \%$. This output mask is defined relatively to the maximum carrier power between $\pm 9 \mathrm{MHz}$. Important points are $-20 \mathrm{dBc}$ at $11 \mathrm{MHz}$ frequency offset from the nominal carrier frequency, $-28 \mathrm{dBC}$ at $20 \mathrm{MHz}$ frequency offset and $-40 \mathrm{dBc}$ above $30 \mathrm{MHz}$ frequency offset. All these points are taken in $1 \mathrm{MHz}$ resolution bandwidth.

To evaluate the impact of desynchronisation between the two paths, simulations of the transmitter were realized on Agilent ADS for a 64 subcarriers OFDM modulation (a worse case in comparison with the 802.11a standard).

Fig. 2 present the impact of the delay for the OFDM modulation on the output spectrum. This delay creates a rotation of the emitted constellation proportional to the subcarrier frequency offset from the nominal carrier frequency and also proportional to the delay itself [3]. For $10 \mathrm{~ns}$, the phase shift can attain 8.5 degrees and EVM is about $30 \%$. For $2 \mathrm{~ns}$, the phase shift is lower than 2 degrees and EVM is about $6.5 \%$. With a time symbol of $50 \mathrm{~ns}$, the maximum acceptable delay is $2 \mathrm{~ns}$, with represents $4 \%$ of the time symbol. This delay can not be achieved without a synchronization algorithm. 


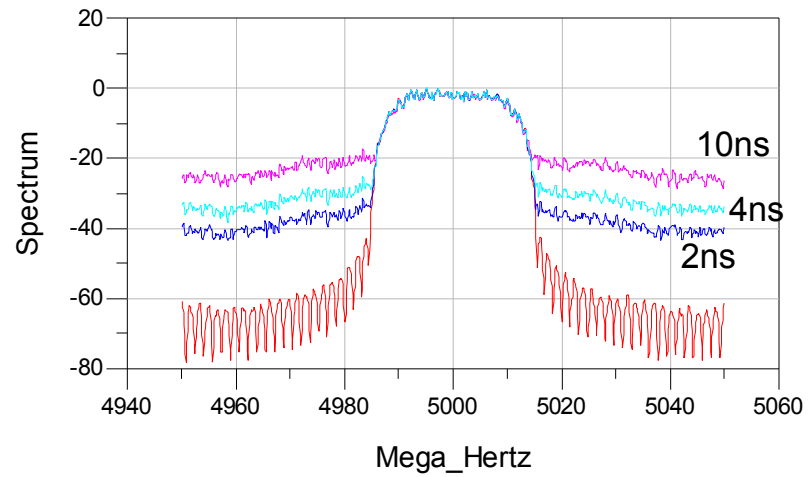

Figure 2. Output spectrum of the OFDM modulation for various delay mismatch: $2 \mathrm{~ns}, 4 \mathrm{~ns}$ and $10 \mathrm{~ns}$

\section{SYNCHRONISATION ALGORITHM}

The EER (Envelope Elimination and Restoration) architecture for digital transmitters enables an efficient use of the power amplifier stage, through the transformation of PA's input $x(t)$ into a constant envelop signal, as was presented in section I. Let us simply consider the in-phase component $x(t)$. This signal $x(t)$ is decomposed into an envelope signal $\rho(t)$, and a phase signal $\cos (\phi(t))$, and the envelope $\rho(t)$ is used as a modulation of PA's supply. But the point is that envelope and phase signals are delayed independently during this processing, and are no more synchronous at the restoration step. Therefore, the theoretical performances of the architecture are degraded. We already reported [3] the degradation of performances due to this time mismatch, in terms of EVM and spectral regrowths. We also proposed [4] a Monte-Carlo evaluation of these envelope/phase delays influences on an OFDM signal and on the overall performance. If we denote by $z_{x}(t)$ the signal at the output of the PA, we have

$$
\left\{\begin{array}{ccc}
x(t) & = & \rho(t) \cos (\phi(t)) \\
z_{x}(t) & = & \rho\left(t-\Delta_{1}\right) \cos \left(\phi\left(t-\Delta_{2}\right)\right)
\end{array}\right.
$$

We propose here to correct the delays using an adaptive precompensation. If one builds a system input signal $\tilde{x}(t)=\rho\left(t+\tau_{1}\right) \cos \left(\phi\left(t+\tau_{2}\right)\right)$, that gives rise to the new output $z_{x}(t)=\rho\left(t+\tau_{1}-\Delta_{1}\right) \cos \left(\phi\left(t+\tau_{2}-\Delta_{2}\right)\right)$, then one has to adjust the delays $\tau_{1}$ and $\tau_{2}$ in order to minimize some (statistical) distance between $\tilde{y}(t)$ and $x(t)$, the minimum being clearly attained for $\tau_{1}=\Delta_{1}$ and $\tau_{2}=\Delta_{2}$. Using this approach, we propose now an adaptive algorithm.

\section{A. Criterion}

A natural criterion is to simply minimize the quadratic distance between $x(t)$ and $z_{x}(t)$ :

$$
J\left(\tau_{1}, \tau_{2}\right)=E\left[\left|x(t)-\rho\left(t_{1}\right) \cos \left(\phi\left(t_{2}\right)\right)\right|^{2}\right],
$$

where we noted, in order to simplify expressions and save space

$$
\left\{\begin{array}{l}
t_{1}=t+\tau_{1}-\Delta_{1} \\
t_{2}=t+\tau_{2}-\Delta_{2}
\end{array}\right.
$$

Note that such a criterion can also be built for the quadrature component, or for both components together. If $z_{y}(t)$ is the output associated to the quadrature component $y(t)=\rho(t) \sin (\phi(t))$, and denoting

$$
\left\{\begin{array}{l}
\underline{x}(t)=[x(t) y(t)]^{t} \\
\underline{z}(t)=\left[z_{x}(t) z_{y}(t)\right]^{t} .
\end{array}\right.
$$

the vectors of the inputs and outputs, and lastly by $\underline{\cos }($. the operator $\underline{\cos }()=.[\cos (.) \sin (.)]^{t}$, the criterion is

$$
J\left(\tau_{1}, \tau_{2}\right)=E\left[\left|\underline{x}(t)-\rho\left(t_{1}\right) \underline{\cos }\left(\phi\left(t_{2}\right)\right)\right|^{2}\right] .
$$

It is important to note that even if criterion (1) has clearly a global minimum for $\tau_{1}=\Delta_{1}$ and $\tau_{2}=\Delta_{2}$, unimodality is not guaranteed and there may exist other (local) minima. It is only in the case of a fixed value $\tau_{1}$ (or $\tau_{2}$ ) that the corresponding criterion has a single minimum.

\section{B. Gradient algorithm}

This criterion can be minimized, with respect to $\tau_{1}$ and $\tau_{2}$, using a gradient algorithm:

$$
\left\{\begin{array}{l}
\tau_{1}(n)=\tau_{1}(n)-\mu_{1} \frac{\partial J\left(\tau_{1}, \tau_{2}\right)}{\partial \tau_{1}} \\
\tau_{2}(n)=\tau_{2}(n)-\mu_{2} \frac{\partial J\left(\tau_{1}, \tau_{2}\right)}{\partial \tau_{2}}
\end{array}\right.
$$

where $\mu_{1}$ and $\mu_{2}$ are two adaptation steps. In our case, the gradients are simply

$$
\frac{\partial J\left(\tau_{1}, \tau_{2}\right)}{\partial \tau_{1}}=-2 E\left[\left.\frac{\mathrm{d} \rho(u)}{\mathrm{d}}\right|_{u=t_{1}} \underline{\cos }\left(\phi\left(t_{2}\right)\right)^{t} \underline{e}(t)\right]
$$

and

$$
\frac{\partial J\left(\tau_{1}, \tau_{2}\right)}{\partial \tau_{2}}=-2 E\left[\left.\frac{\mathrm{d} \phi(u)}{\mathrm{d} u}\right|_{u=t_{2}} \rho\left(t_{1}\right) d \underline{\cos }\left(\phi\left(t_{2}\right)\right)^{t} \underline{e}(t)\right]
$$

with

$$
\underline{e}(t)=\underline{x}(t)-\rho\left(t+\tau_{1}-\Delta_{1}\right) \underline{\cos }\left(\phi\left(t+\tau_{2}-\Delta_{2}\right)\right),
$$

and $d \underline{\cos }()=.[-\sin (.) \cos ()$.$] the derivative of operator$ $\underline{\cos }($.$) . Thus, the update equations are given using these$ gradients in (1). But in practice, we have to resort to an approximation of these theoretical recursions, by adopting a stochastic algorithm.

\section{LMS algorithm}

Hence, we shall use LMS (Least Means Squares) recursions, which simply consist in omitting the statistical mean. Furthermore, iterations of the algorithm and time 
samples are confused, that means that an iteration is done at each time sample. It follows

$$
\left\{\begin{array}{l}
\tau_{1}(n)=\tau_{1}(n)+\left.2 \mu_{1} \frac{\mathrm{d} \rho(u)}{\mathrm{d} u}\right|_{u=t_{1}} \underline{\cos }\left(\phi\left(t_{2}\right)\right)^{t} \underline{e}(t) \\
\tau_{2}(n)=\tau_{2}(n)+\left.2 \mu_{2} \frac{\mathrm{d} \phi(u)}{\mathrm{d} u}\right|_{u=t_{2}} \rho\left(t_{1}\right) d \underline{\cos }\left(\phi\left(t_{2}\right)\right)^{t} \underline{e}(t)
\end{array}\right.
$$

Of course, as such digital processing requires sampling the output of the PA, the available data are on a grid of integer multiples of the sampling period, and $t_{1}$ and $t_{2}$ have a few chances to be and remain on this grid. Therefore, the practical implementation requires proceeding to an interpolation of the signals between time samples.

Different approaches for interpolation can be considered: Shannon-Nyquist ideal interpolation, spheroidal functions, splines... However, experimentations show that a simple interpolation such as a linear interpolation between samples is enough and that the point is not really the quality of interpolation but its presence. In the same vein, the derivatives can be approximated as simple differences between successive samples.

The linear interpolation proceeds as follows. Let $x(k)$ and $x(k+1)$ be two available samples and suppose that one has to estimate $x(k+\Delta k)$, with $\Delta k \in] 0,1[$ then

$$
x(k+\Delta k) \approx \Delta k x(k+1)+(1-\Delta k) x(k) .
$$

This can be easily extended to the case of a delay greater than one.

These points are illustrated by the sample Matlab code, that implements the interpolation of $\cos \phi(t)$ and $\rho(t)$, and the estimation of their derivatives.

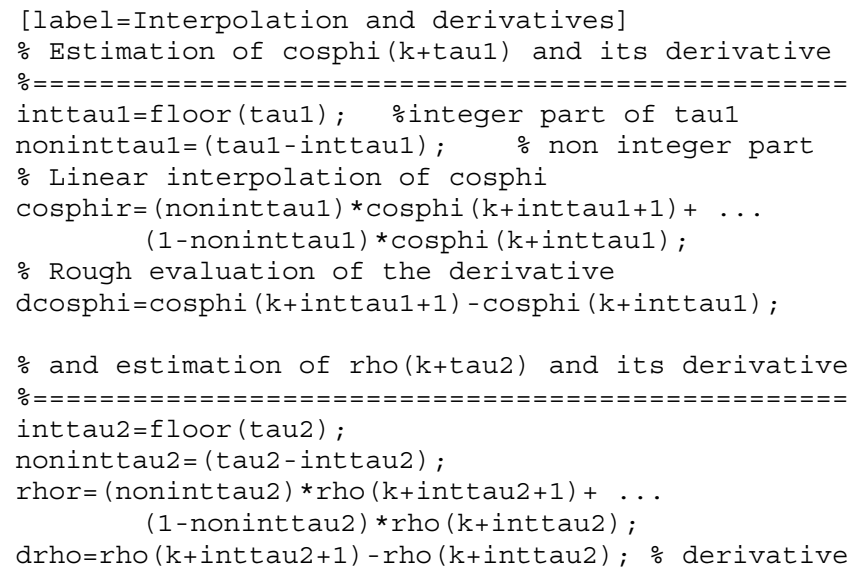

Then the adaptating steps and computation of the restored signal follow immediately. The following code implements (4) and computes the restored signal as :

$$
\underline{x}_{r e s}(t)=\rho\left(t+\tau_{1}-\Delta_{1}\right) \underline{\cos }\left(\phi\left(t+\tau_{2}-\Delta_{2}\right)\right) .
$$

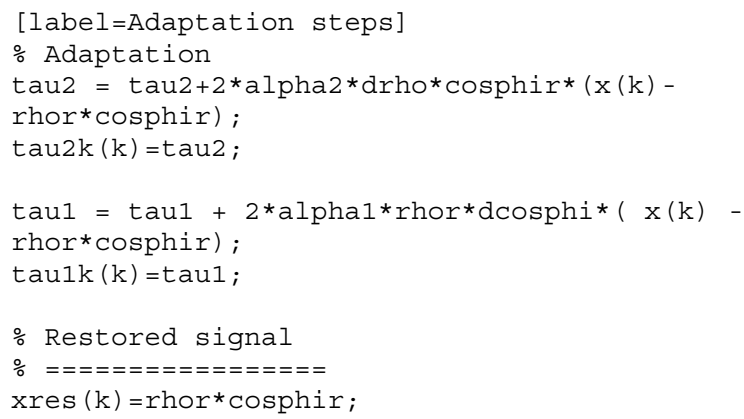

Choice of adaptation steps :

In the case of linear filtering the optimum adaptation step has the form $\mu_{\text {opt }}=2 /\left(\alpha p P_{X}\right)$, where $\alpha$ is a integer between 2 and $4, p$ is the length of the filter and Px the power of the filtered signal. In our case, the problem is not a simple linear filter, but if one consider $\tau_{2}$ fixed, the operation is a simple delay (filtering operation) and as a rule of thumb, one can uses $1 / E\left[\rho^{2}\right]$ as a guideline.

However, it is possible to select these steps automatically using the following trick. It suffices to minimize the criterion $J\left(\tau_{1}, \tau_{2}\right)$, considered as a function of $\tau_{1}$ and $\tau_{2}$, with respect to $\mu_{1}$ and $\mu_{2}$. This can be achieved, again, in an iterative way:

$$
\mu_{i}(n)=\mu_{i}(n-1)-\alpha_{i}\left(J_{\mu_{i}+\Delta \mu_{i}}-J_{\mu_{i}}\right) / \Delta \mu_{i}, i=1,2 .
$$

Of course, so doing, one still has to choose the new parameters $\alpha_{1}$ and $\alpha_{2}$, so that a problem still remains. It also possible to adopt a second order descent method (Newton algorithm).

In our opinion a pragmatical approach that consists in preselecting a set of "good" step corresponding to typical identified situation is also valuable.

\section{Simulation Results}

The adaptive algorithm was implemented with Matlab and with Agilent ADS using Matlab cosimulation. The correction of these delays using the adaptive algorithm presents the adaptive estimation of delays $\tau_{1}$ and $\tau_{2}$ on Fig.3 (where these delays are given as a number of samples). The evolution of the instantaneous error (3) during convergence is presented on Fig.4 and Fig.5 compares the corrected signal to the true and uncorrected ones.

These results are typical of trajectories that can be obtained using our procedure, and show the efficiency and relevance of this approach. In the presented simulation the EVM decrease to $10 \%$ to $3.5 \%$ and power spectrum regrowths are lowered by more than $16 \mathrm{~dB}$ as presented Fig. 6 . 

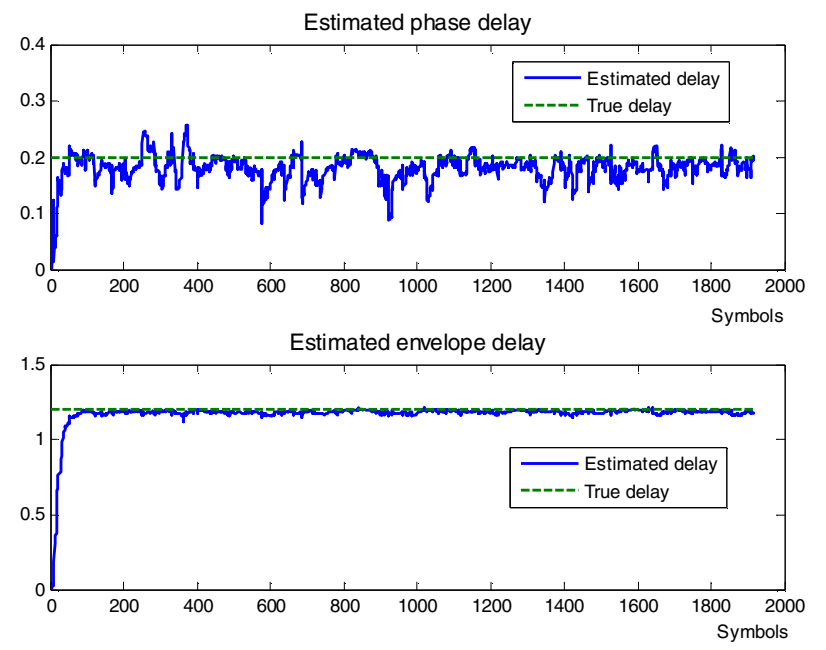

Figure 3. Adaptive estimation of delays $\tau_{1}$ and $\tau_{2}$, given as a fraction of symbol duration.

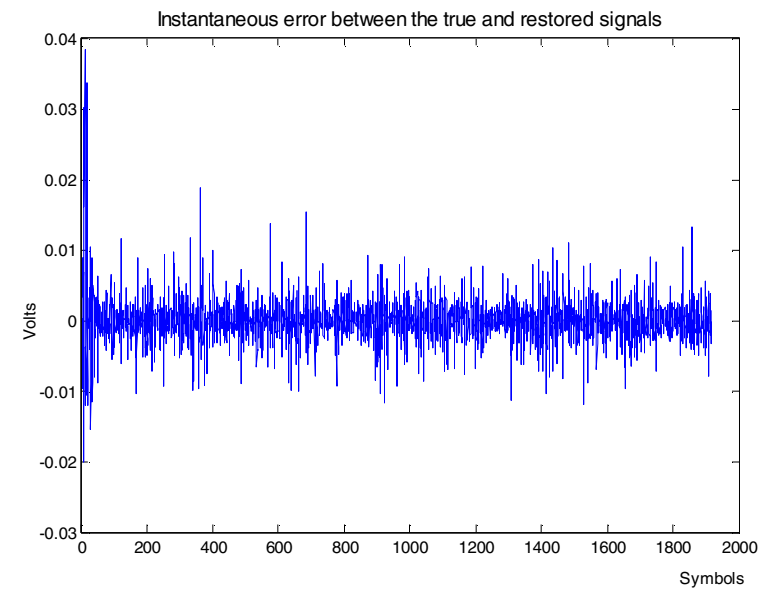

Figure 4. Evolution of the instantaneous error during convergence

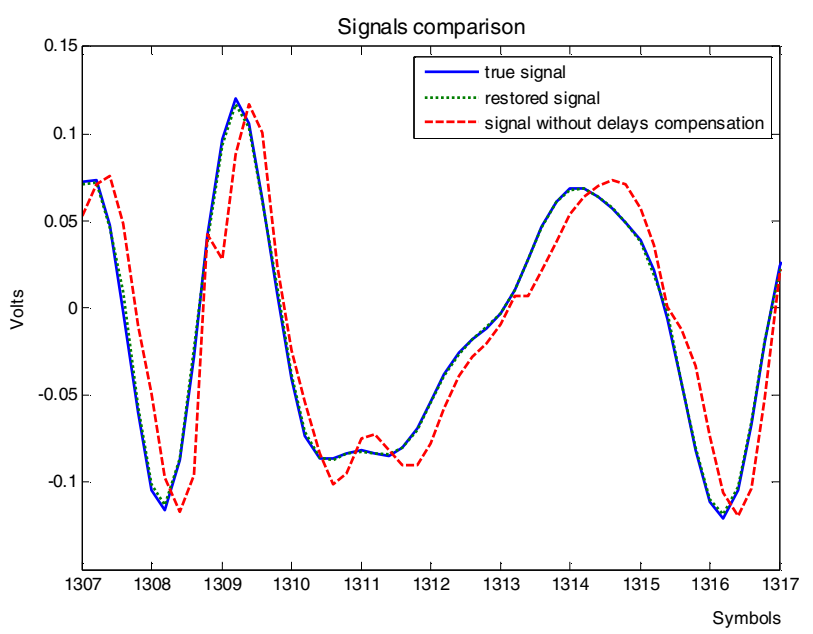

Figure 5. Comparison of the corrected signal to the true and uncorrected ones. The true and uncorrected signals are almost indistinguishable.

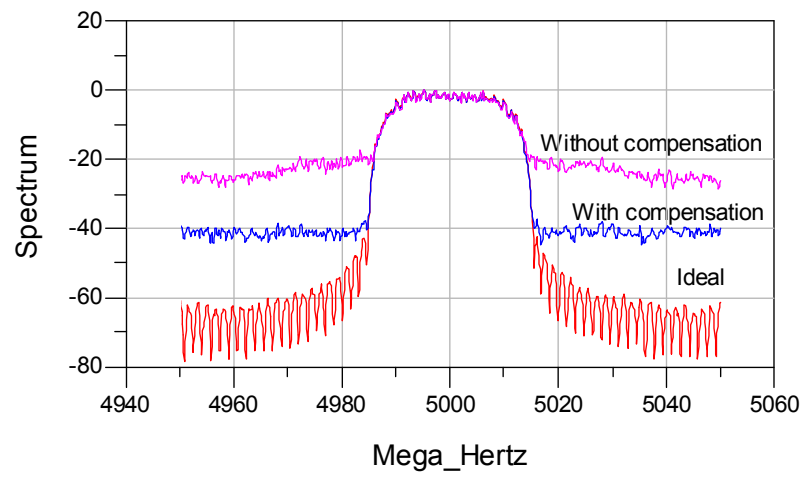

Figure 6. Output spectrum with and without the delay compensation

By reference to Fig.2, we observe that the algorithm clearly enhances the performances, since the output spectrum for a $10 \mathrm{~ns}$ desynchronisation is now similar to a mere $2 \mathrm{~ns}$ desynchronisation. The remaining noise floor is intimately linked to the fluctuations of the estimates (phase and envelope), that in turn, are a function of the values of adaptation steps, as classical with adaptive algorithms.

\section{CONCLUSION}

In this paper, we demonstrate the interest of a compensation principle that dramatically improves the performances in term of EVM and spectrum regrowths of an EER based radio architecture for complex modulations.

Further work includes study of the adaptation steps (notably a decreasing rule of adaptation steps during convergence, that enables the reduction of residual noise). We will also give a precise quantification of improvements and study implementation on physical devices (DSP).

\section{ACKNOWLEDGEMENT}

The authors would like to thank H.F. Dain and B. Miara for many useful discussions and support. We also want to acknowledge the experts who suggested improvements for this communication.

\section{REFERENCES}

[1] L.R.Kahn, "Single sideband transmission by envelope elimination and restoration", proceedings of the I.R.E, 1952, pp.803-806

[2] D.Rudolph, "Kahn EER technique with single carrier digital modulations", IEEE trans on MTT, vol. 51, n², Feb.2003, pp.548552

[3] G. Baudoin, C. Berland, M. Villegas, A. Diet, "Influence of time and processing mismatches between phase and envelope signals in linearization systems using envelope elimination and restoration, application to hiperlan 2", Proc. Conf. IEEE - MTT'2003, Microwave Theory and Technique, June 2003 Philadelphia, USA

[4] JF. Bercher, A. Diet, C. Berland, G. Baudoin, M. Villegas, "Monte Carlo estimation of time mismatch effect in an OFDM EER architecture", IEEE Radio \&Wireless Conference RAWCON 2004, Sept. 2004 